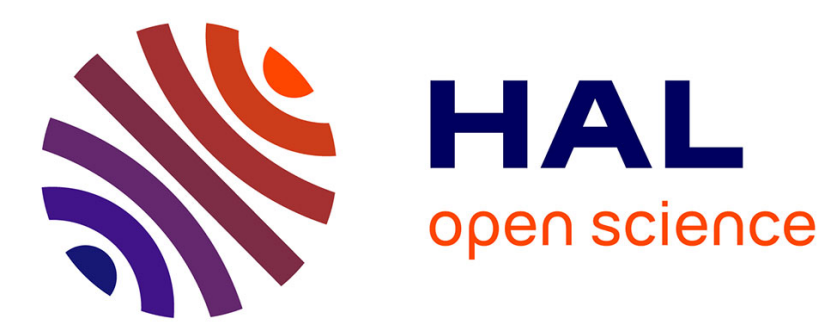

\title{
Etude du comportement et de l'endommagement des composites à matrice aluminium et à renforts particulaires
}

\author{
M. Roger, J. Lu, D. Lai, H. Lieurade
}

\section{- To cite this version:}

M. Roger, J. Lu, D. Lai, H. Lieurade. Etude du comportement et de l'endommagement des composites à matrice aluminium et à renforts particulaires. Journal de Physique IV Proceedings, 1993, 03 (C7), pp.C7-1867-C7-1871. 10.1051/jp4:19937299 . jpa-00251942

HAL Id: jpa-00251942

https://hal.science/jpa-00251942

Submitted on 1 Jan 1993

HAL is a multi-disciplinary open access archive for the deposit and dissemination of scientific research documents, whether they are published or not. The documents may come from teaching and research institutions in France or abroad, or from public or private research centers.
L'archive ouverte pluridisciplinaire HAL, est destinée au dépôt et à la diffusion de documents scientifiques de niveau recherche, publiés ou non, émanant des établissements d'enseignement et de recherche français ou étrangers, des laboratoires publics ou privés. 


\title{
Etude du comportement et de l'endommagement des composites à matrice aluminium et à renforts particulaires
}

\author{
M. ROGER*, J. LU, D. LAI* et H.P. LIEURADE \\ CETIM, BP. 67, 60304 Senlis cedex, France \\ * Université de Technologie de Compiègne, Centre de Recherches de Royallieu, LG2MS, URA 1505 du \\ CNRS, BP. 649, 60206 Compiègne, France
}

\begin{abstract}
Tensile tests were performed on two MMC materials: a recast Duralcan composite $(\mathrm{A} 356+20 \%$ $\mathrm{SiCp})$, and another composite elaborated by powder metallurgy $(2124+15 \% \mathrm{SiCp})$. These composites increased their specific properties such as their Young's modulus, however these improvements were accompanied by a degradation of strain at failure, moreover $\sigma-\varepsilon$ curves were observed with a non linear behavior. In discontinuously reinforced metal matrix composites, microvoid nucleation is the predominant fracture mode. The failure mechanism consist of void nucleation, growth and coalescence stages. The RICE-TRACEY law used to illustrate growth stage was not checked by MMC materials, but WEIBULL's theory was successful; the very low WEIBULL's modulus confirmed the predominant stage of void nucleation by cracking particles. Nucleation of voids is studied numerically. Results of finite element calculation were compared with fracture surfaces. The microscopic observations confirmed the different initiation mechanisms predicted by modeling: cracking for particulate with sharp corner and microvoid in the matrix (A356+20\% SiCp), and debonding for sphere (2124+15\% SiCp). Acoustic emission tests were carried out simultaneously with tensile tests, and showed the damage appearation and his increasement .
\end{abstract}

\section{INTRODUCTION}

L'association d'une matrice métallique et d'une phase dispersée permet d'obtenir de nouvelles propriétés. Nous nous proposons d'étudier un matériau composite élaboré par DURALCAN, puis refondu et moulé par CANMET (Centre Canadien de la Technologie des Minéraux et de l'Energie). Ce composite est constitué d'une matrice en alliage d'aluminium Al-Mg-Si (A356), et de 20\% volumique de renfort particulaire en céramique $(\mathrm{SiC})$.

L'objet de cette étude est de comprendre les mécanismes d'endommagement, afin de pouvoir relier microstructure et propriétés mécaniques en traction monotone et d'utiliser des modèles existants.

Une comparaison avec un second composite permet de mieux apprécier l'influence de différents paramètres, comme le taux et la taille des renforts, sur le mode de rupture. La matrice de ce second composite est un alliage d'aluminium Al-Cu (2124), le taux des renforts en céramique (SiC) est de $15 \%$.

\section{COMPOSITES ETUDIES}

Les propriétés mécaniques de la matrice et des renforts particulaires ainsi que la distribution des particules du CMM A356 sont réunies dans les tableaux ci-dessous [1],[2]:

\begin{tabular}{|c|c|c|c|c|c|c|c|c|}
\hline $\operatorname{Em}(\mathrm{MPa})$ & $v$ & \multicolumn{2}{|c|}{$\operatorname{Re} 0,2 \%(\mathrm{MPa})$} & $\mathrm{Rm}(\mathrm{MPa})$ & A \% & $\mathrm{Ep}(\mathrm{MPa})$ & $v$ & \\
\hline 68600 & 0,3 & \multicolumn{2}{|c|}{230} & 275 & 8 & 450000 & 0,17 & \\
\hline diametre mini & \multicolumn{2}{|c|}{ diametre maxi } & \multicolumn{3}{|c|}{ diamètre moyen des particules } & \multicolumn{3}{|c|}{ distance moyenne entre particules } \\
\hline 2 microns & \multicolumn{2}{|c|}{21 microns } & & \multicolumn{2}{|c|}{15 microns } & \multicolumn{3}{|c|}{23 microns } \\
\hline
\end{tabular}


Le traitement thermique préconisé par CANMET est de type T6. Celui adopté a été optimisé, en collaboration avec le CETIM, par l'EC de LILLE sur le même lot de composite ici étudié [3].

Le CMM 2124 est élaboré par le procédé de la métallurgie des poudres. Les tests mécaniques sont effectués sur des éprouvettes brutes d'élaboration. Les particules de très petite taille (10 fois plus petite que pour le composite A356) sont concentrées autour des grains de poudres initiaux de la matrice. Le tableau suivant donne leur distribution:

\begin{tabular}{|c|c|c|}
\hline diamètre mini & diamètre maxi & diamètre moyen des particules \\
\hline I micron & 4 microns & 3 microns \\
\hline
\end{tabular}

\section{PROPRIETES MECANIQUES SOUS SOLLICITATION MONOTONE [3]}

Les essais de traction ont été effectués sur des éprouvettes parallélépipèdiques de dimensions (partie utile) $30 \times 20 \times 6 \mathrm{~mm}^{3}$ pour le CMM 2124 et $30 \times 10 \times 3 \mathrm{~mm}^{3}$ pour le CMM A356.

* CMM A356

Tableau. Comportement mécanique du CMM A356

\begin{tabular}{|c|c|c|c|c|c|c|c|c|}
\hline $\mathrm{E}(\mathrm{MPa})$ & $\mathrm{v}$ & $\mathrm{Re} 0,2 \%(\mathrm{MPa})$ & $\mathrm{Rm}(\mathrm{MPa})$ & $\varepsilon_{\text {rupt. }} \%$ & $\mathrm{~A} \%$ & $\sigma_{y}(\mathrm{MPa})$ & $\mathrm{K}(\mathrm{MPa})$ & $\mathbf{n}$ \\
\hline 103600 & 0,3 & 309 & 310 & 0,50 & 0,40 & 178 & 3650 & 0,5 \\
\hline
\end{tabular}

Le module de Young est plus élevé que pour le matériau non renforcé. La limite d'élasticité conventionnelle à $0,2 \%$ est très proche de la contrainte à rupture, de même la déformation totale du composite est très faible, d'environ $0,4 \%$. En considérant la limite d'élasticité comme la contrainte maximale avant perte de linéarité de la courbe contrainte-déformation, nous obtenons une valeur $\sigma_{\mathrm{y}}=178$ MPa qui est très faible par rapport à l'alliage non renforcé. La contrainte a rupture est plus élevée que celle de l'alliage non renforcé, mais la déformation à rupture est beaucoup plus faible. Les coefficients d'écrouissage ont été déterminés suivant la loi:

$$
\sigma=\sigma_{\mathrm{y}}+K \varepsilon_{\mathrm{p}}^{\mathrm{n}}
$$

Les caractéristiques du composite ici présenté, sont le résultat d'essais sur une vingtaine d'éprouvettes, la dispersion est importante. Nous pouvons conclure sur un comportement macroscopique de type fragile.

* CMM 2124

Tableau. Comportement mécanicue du CMM 2124

\begin{tabular}{|c|c|c|c|c|c|c|c|c|}
\hline $\mathrm{E}(\mathrm{MPa})$ & $\mathrm{v}$ & $\mathrm{Re} 0,2 \%(\mathrm{MPa})$ & $\mathrm{Rm}(\mathrm{MPa})$ & $\varepsilon_{\text {rupt. }} \%$ & $\mathrm{~A} \%$ & $\sigma_{\mathrm{y}}(\mathrm{MPa})$ & $\mathrm{K}(\mathrm{MPa})$ & $\mathrm{n}$ \\
\hline 90800 & 0,31 & 180 & 290 & 0,7 & 0,60 & 83 & 5600 & 0,7 \\
\hline
\end{tabular}

Nous obtenons une valeur très faible de $\sigma_{\mathrm{y}}$. La déformation à rupture, certes plus élevée que pour le composite précédent, est encore faible. L'écrouissage est plus important que pour le composite moulé alors que le comportement en élasticité montre un composite beaucoup moins rigide. Nous pouvons conclure pour ce composite élaboré par métallurgie des poudres à un comportement macroscopique également de type fragile.

\section{ASPECTS MICROSCOPIQUES ET MECANISMES D'ENDOMMAGEMENT}

Bien que le comportement mécanique soit macroscopiquement fragile, microscopiquement la rupture devrait être ductile. On distingue trois stades dans le processus de rupture ductile[4]:

* nucléation des cavités

* croissance de ces cavités

* coalescence jusqu'à rupture complète du matériau

Il existe trois sites possibles de nucléation [5]:

* dans la matrice, et plus précisément sur les précipités ou sur les inclusions

* à l'interface particule/matrice, par décohésion

* dans les particules par rupture fragile. 
Pour le CMM A356, le faciès type révèle un mélange de presque tous les modes de rupture, mais avec une préférence de ruptures fragiles et de décohésion de particules. Le site de nucléation des cupules se situe à l'interface particule/matrice, et résultent de la décohésion des particules. La nucléation des cavités par décohésion à l'interface particule/matrice est un des mécanismes conduisant à la rupture. Les particules sont assez élancées et de forme anguleuse. Très fragile dans une matrice au contraire beaucoup plus ductile, les angles "vifs" de ces particules se sont rompus sous l'effet de concentration de contraintes qu'ils ont euxmême provoquée. Certaines particules se sont rompues fragilement suivant plusieurs lignes parallèles entre elles, la rupture a pu se faire en traction ou par cisaillement. Le troisième type de rupture de particules est la "Fragmentation". Des particules sont cassées en plusieurs morceaux produisant des cavités relativement importantes dans la matrice[6].

Quant au CMM 2124, les particules, de 3 microns de diamètre, sont beaucoup plus petites que les précédentes (15 microns) et moins anguleuses. La matrice et le procédé de fabrication sont différents. Le faciès de rupture est très peu voisin des précédents. Les particules ne sont pas visibles sur les photos du microscope électronique à balayage. Seule une image RX nous a permis de les identifier comme les petites sphères prédominantes. D'après le faciès, la rupture est relativement ductile, par nucléation puis croissance et coalescence de cupules dans la matrice. Aucune décohésion des particules de SiC n'apparaît, l'interface Matrice/Particule semble très résistante, les particules sont enrobées de matrice (Al). Quelques cupules se sont formées autour de particules de $\mathrm{SiC}$. La rupture dans la matrice est assez surprenante. Une analyse $\mathrm{RX}$ révèle du cuivre et du manganèse[6].

D'une manière générale[5], les mécanismes de rupture dépendent des propriétés de la matrice et de son état métallurgique, de la résistance des particules, de la taille et distribution des particules et des propriétés de l'interface. Soient $\mathrm{f}$ et $\mathrm{d}$ respectivement la fraction volumique et le diamètre moyen des renforts:

- sif augmente, alors $\left(\mathrm{R}_{\mathrm{e}}, \mathrm{R}_{\mathrm{m}}, \mathrm{E}\right)$ augmentent et $\varepsilon_{\mathrm{f}} \%$ diminue

- si d diminue, alors ( $\left.R e, R_{m}, E\right)$ augmentent

et $\quad$ si f augmente $\quad \varepsilon_{\mathrm{f}} \%$ diminue pour un d donné

- si f diminue $\quad \varepsilon_{\mathrm{f}} \%$ augmente pour un donné

\section{MODELISATION}

Approche locale de la rupture - loi de RICE et TRACEY:

RICE et TRACEY proposent un critère de rupture ductile qui est basé sur le mécanisme de croissance et de coalescence des cavités[4]:

$$
\begin{array}{lll}
\ln \frac{\mathrm{R}_{\mathrm{c}}}{\mathrm{R}_{0}}=\int_{\varepsilon_{\text {décohésion }}}^{\varepsilon_{\text {rupture }}} 0,283 \exp \left(1,5 \frac{\sigma_{\mathrm{m}}}{\sigma_{0}}\right) & \mathrm{d} \varepsilon_{\mathrm{oq}}^{\mathrm{p}} \\
& * \mathrm{R} & : \text { rayon de la cavité } \\
\text { avec } & * \mathrm{~d} \varepsilon_{\mathrm{oq}}^{\mathrm{p}} & : \text { incrément de déformation plastique de Von Misés } \\
& * \sigma_{\mathrm{m}} / \sigma_{0} & : \text { taux de triaxialité }(\chi) \\
& * \sigma_{0} & : \text { limite d'élasticité } \\
& * \sigma_{\mathrm{m}} & : \text { contrainte hydrostatique, trace }[\sigma] / 3
\end{array}
$$

\section{Approche statistique de la rupture - loi de WEIBULL (approche globale)}

L' approche statistique considère une probabilité de rupture pour un échantillon fixé à un niveau de contrainte donné. Soient $P_{v}$, la probabilité de rupture d'un volume $V$, WEIBULL propose[7]:

$$
P_{\mathrm{v}}=1-\exp \left[-\left(\frac{\sigma-\sigma_{\mathrm{u}}}{\sigma_{0}}\right)^{\mathrm{m}} \cdot \mathrm{V}\right]
$$

avec $\sigma:$ contrainte appliquée

$\sigma_{0}:$ contrainte de normalisation pour une probabilité de survie de 0,5

$\sigma_{\mathrm{u}}$ : contrainte au-dessous de laquelle il n'y a pas de rupture, contrainte seuil

$\mathrm{m}$ : facteur d'inhomogénéité appelé "module de WEIBULL" 


\section{Modélisation par éléments finis du champ de contraintes autour des particules}

La distribution et la forme des renforts de $\mathrm{SiC}$ jouent un rôle très important dans le mode de rupture du composite. Grâce à un calcul numérique par éléments finis, nous voulons modéliser quelques renforts de formes et de tailles relatives différentes, afin de mieux comprendre localement les causes de différents modes de rupture. Les calculs se font en condition d'axisymétrie, et en élasto-plasticité.

\section{RESULTATS DE MODELISATIONS ET CONCLUSIONS}

Le taux de croissance critique des cavité $\mathrm{R}_{\mathrm{d}} / \mathrm{R}_{0}$ est une constante de matériau. Pour une éprouvette donnée, $\chi$ est également constant. Ainsi $\varepsilon_{\mathrm{eq}}^{\mathrm{p}}$ à rupture doit être, d'après la loi de RICE-TRACEY, une fonction exponentielle de $\chi$. Nous avons effectué des tests en traction monotone sur le CMM A356 pour trois taux de triaxialités: $0,33,0,71$ et 1,10. Les résultats montrés dans la figure 1 ne semblent pas obéir une loi exponentielle. Ceci veut dire que la croissance des cavités, stade d'endommagement très important pour les matériaux élasto-plastiques n'est pas prédominant dans la rupture du CMM étudié.

Les essais de WEIBULL sont effectués sur une vingtaine d'éprouvettes identiques. La probabilité cumulée est calculée comme suit: $P=n /(N+1)$, avec $N$ le nombre total d'essais et $n$ la position d'une éprouvette après les avoir classées de la contrainte à rupture minimale à la contrainte maximale. En mettant la loi de WEIBULL sous forme logarithmique, nous pouvons interpoler les résultats d'essais par une droite de pente $m$ (cf. fig. 2). Pour une valeur de $\sigma_{\mathrm{u}}$ de 250 , nous avons obtenu un $\mathrm{m}$ de 2,56 avec un coefficient de régression de 0,983 . Cette valeur de $m$ est très proche de celle des céramiques. Le bon coefficient de régression et la faible valeur de m montrent que le CMM A356 suit bien la loi de WEIBULL et se comporte comme un matériau fragile. Ceci confirme que la nucléation des cavités est le mode d'endommagement conduisant directement à la rupture du matériau.

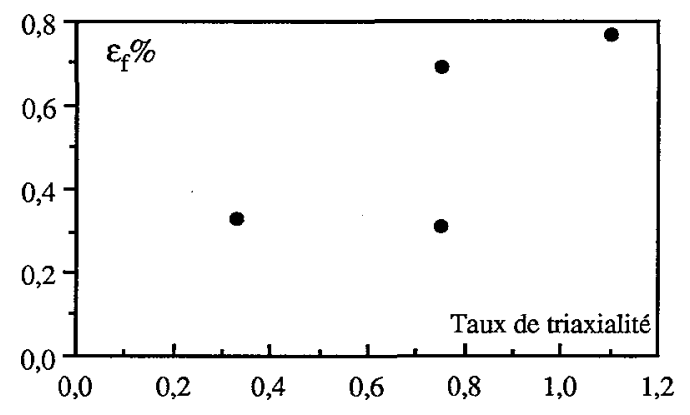

Fig. 1 Courbe $\varepsilon_{\mathrm{f}} \%-\chi$

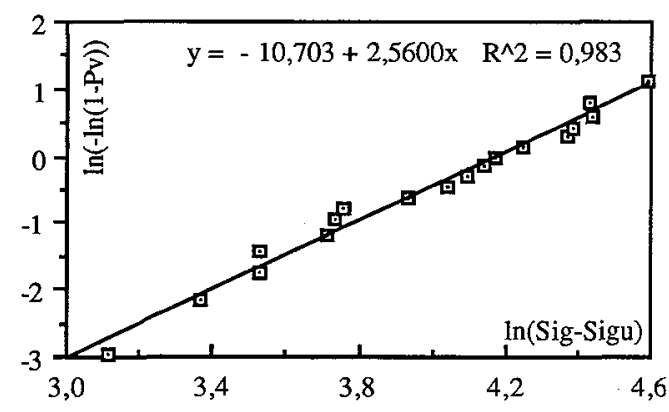

Fig. 2 Courbe logarithmique de WEIBULL

Les résultats de calculs par éléments finis montrent bien que la particule cylindrique, possédant des angles "vifs" (de forme analogue à celle du CMM A356) a des niveaux de contraintes beaucoup plus élevés que la particule sphérique. Ces niveaux de contraintes sont d'autant plus élevés que la taille relative de la particule est grande. La forte concentration de contraintes favorise la rupture ou la décohésion de la particule et donc la nucléation d'une cavité, ce qui est en bon accord avec nos observations expérimentales. 


\section{REFERENCES}

[1] BARRY A., ROUMAGNAC P., CLAVEL M., BERTRAND P., High temperature fatigue behaviour of a cast aluminium alloy, The 3rd International Conference on Aluminium Alloy, Vol. 1, june(1992).

[2] ROGER M., Etude de l'Endommagement de Matériaux Composites à Matrice Métallique, rapport de DEA, Université de Technologie de Compiègne, juillet (1992).

[3] HOTTEBART P., DEGALLAIX S., LIEURADE H.P., LU J., et MEYER, Revue de bibliographie: Composite à Matrice Métallique Al-SiC, Matériaux et Technique, n5 (1993).

[4] SHI Y.W., CHENG J.X. and CHEN B.Y., The Effect of Stress State on the Ductility and the Critical Extent of Void Growth in Nodular Cast Iron, Materials Science and Enginneering, A149(1992) 195-202

[5] MUMMERY P. and DERBY B., The Influence of Microstructure on the Behaviour of Particulate Metal Matrix Composites, Materials Science and Engineering, A135(1991)

[6] ROGER M., LU J., LAI D., CLAVEL M. et LIEURADE H.P., "Etude de mécanismes de rupture de deux composites à matrice métallique (A356+20\% SiCp et $2124+15 \%$ SiCp)", Matériaux et Techniques, $n^{\circ} 5$ (1993).

[7] WEIBULL W., "A statistical distribution function of wide applicability", J. Appl. Mech., 18 (1951) 293-297. 